\title{
Approximation for the Finite-Time Ruin Probability of a General Risk Model with Constant Interest Rate and Extended Negatively Dependent Heavy-Tailed Claims
}

\author{
Yang Yang, ${ }^{1,2}$ Xin $\mathrm{Ma}^{3}$ and Jin-guan Lin $^{2}$ \\ ${ }^{1}$ School of Mathematics and Statistics, Nanjing Audit University, Nanjing 210029, China \\ ${ }^{2}$ Department of Mathematics, Southeast University, Nanjing 210096, China \\ ${ }^{3}$ Golden Audit College, Nanjing Audit University, Nanjing 210029, China
}

Correspondence should be addressed to Yang Yang, yyangmath@gmail.com

Received 6 March 2011; Revised 3 May 2011; Accepted 9 May 2011

Academic Editor: P. Liatsis

Copyright $\odot 2011$ Yang Yang et al. This is an open access article distributed under the Creative Commons Attribution License, which permits unrestricted use, distribution, and reproduction in any medium, provided the original work is properly cited.

We propose a general continuous-time risk model with a constant interest rate. In this model, claims arrive according to an arbitrary counting process, while their sizes have dominantly varying tails and fulfill an extended negative dependence structure. We obtain an asymptotic formula for the finite-time ruin probability, which extends a corresponding result of Wang (2008).

\section{The Dependent General Risk Model}

In this paper, we consider the finite-time ruin probability with constant interest rate in a dependent general risk model. In this model, the claim sizes $\left\{X_{n}, n \geq 1\right\}$ form a sequence of identically distributed, not necessarily independent, and nonnegative random variables (r.v.s) with common distribution $F$ such that $\bar{F}(x)=1-F(x)=\mathrm{P}\left(X_{1}>x\right)>0$ for all $x>0$; the claim arrival process $\{N(t), t \geq 0\}$ is a general counting process, namely, a nonnegative, nondecreasing, right continuous, and integer-valued stochastic process with $0<\mathrm{EN}(t)=$ $\lambda(t)<\infty$ for all large $t>0$. The times of the successive claims are denoted by $\left\{\tau_{n}, n \geq 1\right\}$. The total amount of premiums accumulated up to time $t \geq 0$, denoted by $C(t)$ with $C(0)=0$ and $C(t)<\infty$ almost surely for every $t>0$, is another nonnegative and nondecreasing stochastic process. Assume that $\left\{X_{n}, n \geq 1\right\},\{N(t), t \geq 0\}$ and $\{C(t), t \geq 0\}$ are mutually independent. Let $\delta>0$ be the constant interest rate (i.e., after time $t$ one dollar becomes $e^{\delta t}$ dollars), and 
let $x \geq 0$ be the initial capital reserve of an insurance company. Then, the total discounted reserve up to time $t \geq 0$, denoted by $D(t, x)$, can be written as

$$
D(t, x)=x+\int_{0}^{t} e^{-\delta s} C(\mathrm{~d} s)-\sum_{n=1}^{N(t)} X_{n} e^{-\delta \tau_{n}}
$$

For a finite time $T>0$, the finite-time ruin probability is defined by

$$
\begin{aligned}
\Psi(x, T) & =\mathrm{P}(D(t, x)<0, \text { for some } 0 \leq t \leq T) \\
& =\mathrm{P}\left(\sup _{t \in[0, T]}\left(\sum_{n=1}^{N(t)} X_{n} e^{-\delta \tau_{n}}-\int_{0}^{t} e^{-\delta s} C(\mathrm{~d} s)\right)>x\right),
\end{aligned}
$$

while the ultimate ruin probability is defined by

$$
\Psi(x)=\Psi(x, \infty)=\mathrm{P}(D(t, x)<0, \text { for some } t \geq 0) .
$$

If the claim sizes $\left\{X_{n}, n \geq 1\right\}$ are independent r.v.s, the model is called the independent general risk model, which was introduced by Wang [1]. In particular, if $C(t)=c t, t \geq 0$, with $c>0$ a deterministic constant and $\{N(t), t \geq 0\}$ is a Poisson process, then the model reduces to the classical one.

\section{Introduction and Main Result}

Hereafter, all limit relationships hold for $x$ tending to $\infty$ unless otherwise stated. For two positive functions $f(x)$ and $g(x)$, we write $f(x) \sim g(x)$ if $\lim f(x) / g(x)=1$; write $f(x) \lesssim$ $g(x)$ if $\limsup f(x) / g(x) \leq 1$ and $f(x)=o(g(x))$ if $\lim f(x) / g(x)=0$. The indicator function of an event $A$ is denoted by $\mathbf{1}_{A}$.

In risk theory, heavy-tailed distributions are often used to model large claim amounts. They play a key role in insurance and finance. We will restrict the claim-size distribution $F$ to be heavy tailed. A distribution $V$ is said to be dominatedly varying tailed, denoted by $V \in \Phi$, if limsup $\bar{V}(x y) / \bar{V}(x)<\infty$ for any $y>0$. A distribution $V$ is said to be long tailed, denoted by $V \in \mathcal{L}$, if $\lim \bar{V}(x+y) / \bar{V}(x)=1$ for any $y>0$. A distribution $V$ is said to be subexponential, denoted by $V \in \mathcal{S}$, if $\overline{V^{n *}}(x) \sim n \bar{V}(x)$ for any $n \geq 2$, where $V^{n *}$ denotes the $n$-fold convolution of itself. A distribution $V$ is said to be regularly varying tailed, denoted by $\mathcal{R}_{-\alpha}, \alpha>0$, if $\lim \bar{V}(x y) / \bar{V}(x)=y^{-\alpha}$ for any $y \geq 1$. A proper inclusion relationship holds that

$$
R_{-\alpha} \subset \mathcal{L} \cap \Phi \subset S \subset \mathcal{L}
$$


see, for example, Cline [2] or Embrechts and Omey [3]. For a distribution $V$, denote the upper Matuszewska index of the distribution $V$ by

$$
J_{V}^{+}=-\lim _{y \rightarrow \infty} \frac{\log \bar{V}_{*}(y)}{\log y} \quad \text { with } \bar{V}_{*}(y)=\liminf _{x \rightarrow \infty} \frac{\bar{V}(x y)}{\bar{V}(x)}, \quad y>1 .
$$

In the terminology of Bingham et al. [4], the quantity $J_{V}^{+}$is actually the upper Matuszewska index of the function $1 / \bar{V}(x), x \geq 0$, as also pointed out in Tang and Tsitsiashvili [5]. Additionally, denote $L_{V}=\lim _{y \backslash 1} \bar{V}_{*}(y)$ (clearly, $0 \leq L_{V} \leq 1$ ). The presented definitions yield that the following assertions are equivalent:
(i) $V \in \Phi$,
(ii) $\bar{V}_{*}(y)>0$ for some $y>1$,
(iii) $L_{V}>0$,
(iv) $J_{V}^{+}<\infty$.

The asymptotic behavior of the ruin probability in the classical risk model has been extensively investigated in the literature. Klüppelberg and Stadtmüller [6] considered the ultimate ruin probability for the case of regularly-varying-tailed claim sizes. Using the reflected random walk theory, Asmussen [7] extended the study to a larger class of heavy-tailed distributions; see Corollary 4.1(ii) of his paper. Complementary discussions on the ultimate ruin probability can be found in Kalashnikov and Konstantinides [8], Konstantinides et al. [9], Tang [10], among others.

In this paper, we are interested in the finite-time ruin probability. In this aspect, Tang [11] established an asymptotic result in the classical risk model: under the condition $F \in \mathcal{S}$, he obtained that for every $T>0$ for which $\lambda(T)>0$,

$$
\Psi(x, T) \sim \int_{0-}^{T} \bar{F}\left(x e^{\delta t}\right) \lambda(\mathrm{d} t)
$$

Recently, Wang [1] derived some important and interesting results in two independent risk models. One is the delayed renewal risk model, in which (2.4) holds if $F \in \mathcal{S}$; another is the

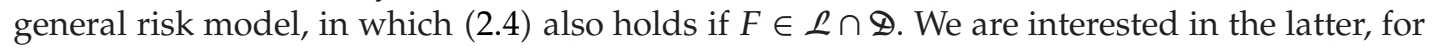
example, the general risk model, and restate Theorem 2.2 of Wang [1] here.

Theorem 2.1. In the independent general risk model introduced in Section 1, assume that the claim sizes $\left\{X_{n}, n \geq 1\right\}$ are independent and identically distributed nonnegative r.v.s with common distribution $F \in \mathcal{L} \cap \boldsymbol{\Phi}$. Assume that for any $T>0$ with $\lambda(T)-\lambda(0)>0$, there exists some constant $\eta=\eta(T)>0$ such that

$$
\mathrm{E}(1+\eta)^{N(T)}<\infty
$$

Then, (2.4) holds.

In the present paper, we aim to deal with the extended negatively dependent general risk model to get a similar result under $F \in \boldsymbol{D}$. Simultaneously, the condition (2.5) can be weakened to $(2.8)$ below. 
We call r.v.s $\left\{\xi_{n}, n \geq 1\right\}$ are extended negatively dependent (END) if there exists some positive constant $M$ such that both

$$
\begin{aligned}
& \mathrm{P}\left(\bigcap_{k=1}^{n}\left\{\xi_{k}>y_{k}\right\}\right) \leq M \prod_{k=1}^{n} \mathrm{P}\left(\xi_{k}>y_{k}\right), \\
& \mathrm{P}\left(\bigcap_{k=1}^{n}\left\{\xi_{k} \leq y_{k}\right\}\right) \leq M \prod_{k=1}^{n} \mathrm{P}\left(\xi_{k} \leq y_{k}\right)
\end{aligned}
$$

hold for each $n \geq 1$ and all $y_{1}, \ldots, y_{n}$. This dependence structure was introduced by Liu [12]. Recall that r.v.s $\left\{\xi_{n}, n \geq 1\right\}$ are called upper negatively dependent (UND) if (2.6) holds with $M=1$, they are called lower negatively dependent (LND) if (2.7) holds with $M=1$, and they are called negatively dependent (ND) if both (2.6) and (2.7) hold with $M=1$. These negative dependence structures were introduced by Ebrahimi and Ghosh [13] and Block et al. [14]. Clearly, ND r.v.s must be END r.v.s., and Example 4.1 of Liu [12] shows that the END structure also includes some other dependence structures.

Motivated by the work of Wang [1], under the END structure, we formulate our main result as follows.

Theorem 2.2. In the dependent general risk model introduced in Section 1, assume that the claim sizes $\left\{X_{n}, n \geq 1\right\}$ are END nonnegative r.v.s with common distribution $F \in \Phi$ and finite mean $\mu$. Assume that for any $T>0$ with $\lambda(T)-\lambda(0)>0$, there exists some constant $p>J_{F}^{+}$such that

$$
\mathrm{E}(N(T))^{p}<\infty .
$$

Then, it holds that

$$
L_{F} \int_{0-}^{T} \bar{F}\left(x e^{\delta t}\right) \lambda(d t) \lesssim \Psi(x, T) \lesssim L_{F}^{-1} \int_{0-}^{T} \bar{F}\left(x e^{\delta t}\right) \lambda(d t)
$$

Furthermore, if $F \in \mathcal{L} \cap \Phi$, then (2.4) holds.

The rest of the present paper consists of two sections. We give some lemmas and the proof of Theorem 2.2 in Section 3. In Section 4, we perform some numerical calculations to verify the approximate relationship in our main result.

\section{Proof of Main Result and Some Lemmas}

In the sequel, $M$ and $a$ always represent some finite and positive constants whose values may vary in different places. In this section, we start by giving some lemmas to show some properties of the class $\Phi$ and the END structure. The first lemma is a combination of Proposition 2.2.1 of Bingham et al. [4] and Lemma 3.5 of Tang and Tsitsiashvili [15].

Lemma 3.1. If a distribution $V \in \boldsymbol{\Phi}$, then

(i) for any $r>J_{V}^{+}$, there exist positive constants $a$ and $b$ such that $\bar{V}(y) / \bar{V}(x) \leq a(y / x)^{-\gamma}$ holds for all $x \geq y \geq b$ and

(ii) it holds for every $\gamma>J_{V}^{+}$that $x^{-\gamma}=o(\bar{V}(x))$. 
By direct verification, END r.v.s have the following properties similar to those of ND r.v.s; see Lemma 3.1 of Liu [12]. For some refined properties of END r.v.s, one can refer to Chen et al. [16]. The following lemma can also be found in Lemma 2.2 of Chen et al. [16].

Lemma 3.2. (i) If r.v.s $\left\{\xi_{n}, n \geq 1\right\}$ are nonnegative and $E N D$, then for any $n \geq 1$, there exists some positive constant $M$ such that $\mathrm{E}\left(\prod_{k=1}^{n} \xi_{k}\right) \leq M \prod_{k=1}^{n} \mathrm{E} \xi_{k}$.

(ii) If r.v.s $\left\{\xi_{n}, n \geq 1\right\}$ are END and $\left\{f_{n}(\cdot), n \geq 1\right\}$ are either all monotone increasing or all monotone decreasing, then $\left\{f_{n}\left(\xi_{n}\right), n \geq 1\right\}$ are still END.

The following two lemmas play important roles in the proof of our main result.

Lemma 3.3. Let $\left\{\xi_{n}, n \geq 1\right\}$ be identically distributed and END r.v.s with common distribution $V$ and $\mu_{V}^{+}=\mathrm{E} \xi_{1} \mathbf{1}_{\left\{\xi_{1} \geq 0\right\}}<\infty$. Then, for any $\theta>0, x>0$ and $n \geq 1$, there exists some positive constant $M$ such that

$$
\mathrm{P}\left(\sum_{k=1}^{n} \xi_{k}>x\right) \leq n \bar{V}(\theta x)+M\left(\frac{e \mu_{V}^{+} n}{x}\right)^{\theta^{-1}}
$$

Proof. Following the proof of Lemma 2.3 of Tang [17], we employ a standard truncation argument to prove this lemma. For simplicity, we write $S_{n}^{\xi}=\sum_{k=1}^{n} \xi_{k}, n \geq 1$. If $\mu_{V}^{+}=0$, then $\xi_{n}$ is almost surely nonpositive for each $n \geq 1$, implying $\mathrm{P}\left(S_{n}^{\xi}>x\right)=0$ for any positive $x$, and thus (3.1) holds.

Let, in the following, $\mu_{V}^{+}>0$. For any fixed $\theta>0$ and positive integer $n$, define

$$
\begin{aligned}
& \tilde{\xi}_{n}=\min \left\{\xi_{n}, \theta x\right\} \\
& \tilde{\xi}_{n}^{+}=\max \left\{\tilde{\xi}_{n}, 0\right\}=\xi_{n} \mathbf{1}_{\left\{0 \leq \xi_{n} \leq \theta x\right\}}+\theta x \mathbf{1}_{\left\{\xi_{n}>\theta x\right\}} .
\end{aligned}
$$

According to Lemma 3.2(ii), $\left\{\tilde{\xi}_{n}, n \geq 1\right\}$ and $\left\{\tilde{\xi}_{n}^{+}, n \geq 1\right\}$ are still END r.v.s, respectively. Denote $\widetilde{S}_{n}^{\xi}=\sum_{k=1}^{n} \widetilde{\xi}_{k}, n \geq 1$. Clearly,

$$
\begin{aligned}
\mathrm{P}\left(S_{n}^{\xi}>x\right) & =\mathrm{P}\left(S_{n}^{\xi}>x, \max _{1 \leq k \leq n} \xi_{k}>\theta x\right)+\mathrm{P}\left(S_{n}^{\xi}>x, \max _{1 \leq k \leq n} \xi_{k} \leq \theta x\right) \\
& \leq n \bar{V}(\theta x)+\mathrm{P}\left(\widetilde{S}_{n}^{\xi}>x\right) .
\end{aligned}
$$

It remains to estimate the second summand in (3.3). For a positive $h$, by Lemma 3.2(ii), $\left\{e^{h \tilde{\xi}_{n}^{+}}, n \geq 1\right\}$ are END nonnegative r.v.s. Hence, using identity

$$
\mathrm{E} e^{h \tilde{\xi}_{1}^{+}}=\int_{0}^{\theta x}\left(e^{h u}-1\right) V(\mathrm{~d} u)+\left(e^{h \theta x}-1\right) \bar{V}(\theta x)+1
$$


by Markov inequality and Lemma 3.2(i) we have

$$
\begin{aligned}
\mathrm{P}\left(\widetilde{S}_{n}^{\xi}>x\right) & \leq e^{-h x} \mathrm{E}^{h \widetilde{S}_{n}^{\xi}} \\
& \leq e^{-h x} \mathrm{E}^{h \sum_{k=1}^{n} \tilde{\xi}_{k}^{+}} \\
& \leq e^{-h x} M\left(\mathrm{E}^{h \tilde{\xi}_{1}^{+}}\right)^{n} \\
& =M e^{-h x}\left(\int_{0}^{\theta x}\left(e^{h u}-1\right) V(\mathrm{~d} u)+\left(e^{h \theta x}-1\right) \bar{V}(\theta x)+1\right)^{n}
\end{aligned}
$$

Since $1+u \leq e^{u}$ for all $u \in \mathbb{R}$ and $\left(e^{h u}-1\right) / u$ is strictly increasing in $u>0$, from (3.5), we obtain

$$
\begin{aligned}
\mathrm{P}\left(\widetilde{S}_{n}^{\xi}>x\right) & \leq M \exp \left\{n \int_{0}^{\theta x} \frac{e^{h u}-1}{u} u V(\mathrm{~d} u)+n\left(e^{h \theta x}-1\right) \bar{V}(\theta x)-h x\right\} \\
& \leq M \exp \left\{n \frac{e^{h \theta x}-1}{\theta x}\left(\int_{0}^{\theta x} u V(\mathrm{~d} u)+\theta x \bar{V}(\theta x)\right)-h x\right\} \\
& \leq M \exp \left\{n \frac{e^{h \theta x}-1}{\theta x} \mu_{V}^{+}-h x\right\} .
\end{aligned}
$$

Choose $h=(\theta x)^{-1} \log \left(x\left(\mu_{V}^{+} n\right)^{-1}+1\right)$, which is positive. For such $h$, by (3.6), we have

$$
\begin{aligned}
\mathrm{P}\left(\widetilde{S}_{n}^{\xi}>x\right) & \leq M \exp \left\{\frac{1}{\theta}-\frac{1}{\theta} \log \left(\frac{x}{\mu_{V}^{+} n}+1\right)\right\} \\
& \leq M \exp \left\{\frac{1}{\theta} \log \frac{e \mu_{V}^{+} n}{x}\right\} .
\end{aligned}
$$

The last estimate and (3.3) imply the desired estimate (3.1). The lemma is proved.

Lemma 3.4. In the dependent general risk model introduced in Section 1, assume that the claim sizes $\left\{X_{n}, n \geq 1\right\}$ are END nonnegative r.v.s with common distribution $F \in \Phi$. Let $Z$ be an arbitrary nonnegative r.v. and assume that $\left\{X_{n}, n \geq 1\right\},\{N(t), t \geq 0\}$ and $Z$ are mutually independent. Then, for any $T>0$ and any positive integer $n_{0}$,

$$
\begin{aligned}
L_{F} \sum_{k=1}^{n_{0}} \sum_{j=1}^{k} \mathrm{P}\left(X_{j} e^{-\delta \tau_{j}}>x, N(T)=k\right) & \lesssim \sum_{k=1}^{n_{0}} \mathrm{P}\left(\sum_{j=1}^{k} X_{j} e^{-\delta \tau_{j}}>x+Z, N(T)=k\right) \\
& \lesssim L_{F}^{-1} \sum_{k=1}^{n_{0}} \sum_{j=1}^{k} \mathrm{P}\left(X_{j} e^{-\delta \tau_{j}}>x, N(T)=k\right) .
\end{aligned}
$$


Furthermore, if $F \in \mathcal{L} \cap \Phi$, then

$$
\sum_{k=1}^{n_{0}} P\left(\sum_{j=1}^{k} X_{j} e^{-\delta \tau_{j}}>x+Z, N(T)=k\right) \sim \sum_{k=1}^{n_{0}} \sum_{j=1}^{k} P\left(X_{j} e^{-\delta \tau_{j}}>x, N(T)=k\right) .
$$

We remark that if $F$ is consistently varying tailed (see the definition in Chen and Yuen [18]), then by conditioning (3.9) easily follows from Theorem 3.2 of Chen and Yuen [18]. Note that this case is in a broader scope, since there is no need to assume independence between $\left(\tau_{1}, \ldots, \tau_{n_{0}}\right)$ and $Z$.

Proof. We follow the line of the proof of Lemma 3.6 of Wang [1] with some modifications in relation to the properties of the class $\Phi$ and the END structure. Clearly, for each $k=1, \ldots, n_{0}$,

$$
\begin{aligned}
& \mathrm{P}\left(\sum_{j=1}^{k} X_{j} e^{-\delta \tau_{j}}>x+Z, N(T)=k\right) \\
& \quad=\int_{\left\{0 \leq t_{1} \leq \cdots \leq t_{k} \leq T, t_{k+1}>T\right\}} \int_{0-}^{\infty} \mathrm{P}\left(\sum_{j=1}^{k} X_{j} e^{-\delta t_{j}}>x+z\right) \times \mathrm{P}\left(Z \in \mathrm{d} z, \tau_{1} \in \mathrm{d} t_{1}, \ldots, \tau_{k+1} \in \mathrm{d} t_{k+1}\right) .
\end{aligned}
$$

We first show the upper bound. For any fixed $l>0$,

$$
\begin{aligned}
\mathrm{P}\left(\sum_{j=1}^{k} X_{j} e^{-\delta t_{j}}>x+z\right) \leq & \mathrm{P}\left(\bigcup_{j=1}^{k}\left\{X_{j} e^{-\delta t_{j}}>x+z-l\right\}\right) \\
& +\mathrm{P}\left(\sum_{j=1}^{k} X_{j} e^{-\delta t_{j}}>x+z, \max _{1 \leq j \leq k} X_{j} e^{-\delta t_{j}} \leq x+z-l\right):=I_{1}+I_{2} .
\end{aligned}
$$

By $F \in \Phi$, for any $0<\theta<1$ and each $k=1, \ldots, n_{0}$, we have uniformly for all $t_{1}, \ldots, t_{k} \in[0, T]$ and $z \in[0, \infty)$,

$$
I_{1} \leq \sum_{j=1}^{k} \bar{F}\left(\theta(x+z) e^{\delta t_{j}}\right) \lesssim L_{F}^{-1} \sum_{j=1}^{k} \bar{F}\left((x+z) e^{\delta t_{j}}\right)
$$


by firstly letting $x \rightarrow \infty$ then $\theta \nearrow 1$. We note that $\left\{X_{n}, n \geq 1\right\}$ are END r.v.s. Then, by $F \in \Phi$, there exists some positive constant $M=M\left(n_{0}\right)$ such that for sufficiently large $x$, each $k=1, \ldots, n_{0}$, all $t_{1}, \ldots, t_{k} \in[0, T]$ and $z \in[0, \infty)$,

$$
\begin{aligned}
I_{2} & =\mathrm{P}\left(\sum_{j=1}^{k} X_{j} e^{-\delta t_{j}}>x+z, \frac{x+z}{k}<\max _{1 \leq j \leq k} X_{j} e^{-\delta t_{j}} \leq x+z-l\right) \\
& \leq \mathrm{P}\left(\bigcup_{i=1}^{k}\left\{\sum_{j \neq i} X_{j} e^{-\delta t_{j}}>l, X_{i} e^{-\delta t_{i}}>\frac{x+z}{k}\right\}\right) \\
& \leq \sum_{i=1}^{k} \sum_{j \neq i} \mathrm{P}\left(X_{j} e^{-\delta t_{j}}>\frac{l}{k-1}, X_{i} e^{-\delta t_{i}}>\frac{x+z}{k}\right) \\
& \leq M \sum_{i=1}^{k} \sum_{j \neq i} \bar{F}\left(\frac{l e^{\delta t_{j}}}{k-1}\right) \bar{F}\left(\frac{(x+z) e^{\delta t_{i}}}{k}\right) \\
& \leq M \bar{F}\left(\frac{l}{n_{0}-1}\right) \sum_{j=1}^{k} \bar{F}\left((x+z) e^{\delta t_{j}}\right) .
\end{aligned}
$$

Since $l$ can be arbitrarily large, it follows that

$$
\limsup _{l \rightarrow \infty} \limsup _{x \rightarrow \infty} \sup _{t_{1}, \ldots, t_{k} \in[0, T], z \in[0, \infty)} \frac{I_{2}}{\sum_{j=1}^{k} \bar{F}\left((x+z) e^{\delta t_{j}}\right)}=0 .
$$

Hence, from (3.10)-(3.14), we obtain for each $k=1, \ldots, n_{0}$,

$$
\begin{aligned}
\mathrm{P}\left(\sum_{j=1}^{k} X_{j} e^{-\delta \tau_{j}}>x+Z, N(T)=k\right) \lesssim & L_{F}^{-1} \sum_{j=1}^{k} \int_{\left\{0 \leq t_{1} \leq \cdots \leq t_{k} \leq T, t_{k+1}>T\right\}} \int_{0-}^{\infty} \bar{F}\left((x+z) e^{\delta t_{j}}\right) \\
& \times \mathrm{P}\left(Z \in \mathrm{d} z, \tau_{1} \in \mathrm{d} t_{1}, \ldots, \tau_{k+1} \in \mathrm{d} t_{k+1}\right) \\
= & L_{F}^{-1} \sum_{j=1}^{k} \mathrm{P}\left(X_{j} e^{-\delta \tau_{j}}>x+Z, N(T)=k\right) \\
\leq & L_{F}^{-1} \sum_{j=1}^{k} \mathrm{P}\left(X_{j} e^{-\delta \tau_{j}}>x, N(T)=k\right) .
\end{aligned}
$$


As for the lower bound for (3.10), since $\left\{X_{n}, n \geq 1\right\}$ are END r.v.s, we have for sufficiently large $x$ and each $k=1, \ldots, n_{0}$,

$$
\begin{aligned}
\mathrm{P}\left(\sum_{j=1}^{k} X_{j} e^{-\delta t_{j}}>x+z\right) & \geq \mathrm{P}\left(\bigcup_{j=1}^{k}\left\{X_{j} e^{-\delta t_{j}}>x+z\right\}\right) \\
& \geq \sum_{j=1}^{k} \bar{F}\left((x+z) e^{\delta t_{j}}\right)-\sum_{1 \leq i<j \leq k} \mathrm{P}\left(X_{i} e^{-\delta t_{i}}>x+z, X_{j} e^{-\delta t_{j}}>x+z\right) \\
& \geq \sum_{j=1}^{k} \bar{F}\left((x+z) e^{\delta t_{j}}\right)-M \sum_{1 \leq i<j \leq k} \bar{F}\left((x+z) e^{\delta t_{i}}\right) \bar{F}\left((x+z) e^{\delta t_{j}}\right) \\
& =(1-o(1)) \sum_{j=1}^{k} \bar{F}\left((x+z) e^{r t_{j}}\right)
\end{aligned}
$$

holds uniformly for all $t_{1}, \ldots, t_{k} \in[0, T]$ and $z \in[0, \infty)$. By $F \in \Phi$ and Fatou's lemma, we have for any $\tilde{\theta}>1$ and all $j=1,2, \ldots$,

$$
\begin{aligned}
\liminf \frac{1}{\bar{F}(x)} \mathrm{P}\left(X_{j}>x+Z e^{\delta T}\right) & =\liminf \int_{0-}^{\infty} \frac{\bar{F}\left(x+z e^{\delta T}\right)}{\bar{F}(x)} \mathrm{P}(Z \in \mathrm{d} z) \\
& \geq \int_{0-}^{\infty} \liminf \frac{\bar{F}(\tilde{\theta} x)}{\bar{F}(x)} \mathrm{P}(Z \in \mathrm{d} z) \\
& =\overline{F_{*}}(\tilde{\theta}) \longrightarrow L_{F}, \quad \tilde{\theta} \searrow 1,
\end{aligned}
$$

which means

$$
\mathrm{P}\left(X_{j}>x+Z e^{\delta T}\right) \gtrsim L_{F} \bar{F}(x)
$$

Similar to (3.15), from (3.10), (3.16), and (3.18), we obtain for each $k=1, \ldots, n_{0}$,

$$
\begin{aligned}
\mathrm{P}\left(\sum_{j=1}^{k} X_{j} e^{-\delta \tau_{j}}>x+Z, N(T)=k\right) \\
\gtrsim \sum_{j=1}^{k} \mathrm{P}\left(X_{j} e^{-\delta \tau_{j}}>x+Z, N(T)=k\right)
\end{aligned}
$$




$$
\begin{aligned}
& \geq \sum_{j=1}^{k} \int_{\left\{0 \leq t_{1} \leq \ldots \leq t_{k} \leq T, t_{k+1}>T\right\}} \mathrm{P}\left(\mathrm{X}_{j}>x e^{\delta t_{j}}+Z e^{\delta T}\right) \mathrm{P}\left(\tau_{1} \in \mathrm{d} t_{1}, \ldots, \tau_{k+1} \in \mathrm{d} t_{k+1}\right) \\
& \gtrsim L_{F} \sum_{j=1}^{k} \int_{\left\{0 \leq t_{1} \leq \ldots \leq t_{k} \leq T, t_{k+1}>T\right\}} \bar{F}\left(x e^{\delta t_{j}}\right) \mathrm{P}\left(\tau_{1} \in \mathrm{d} t_{1}, \ldots, \tau_{k+1} \in \mathrm{d} t_{k+1}\right) \\
& =L_{F} \sum_{j=1}^{k} \mathrm{P}\left(X_{j} e^{-\delta \tau_{j}}>x, N(T)=k\right) .
\end{aligned}
$$

The desired relation (3.8) follows now from (3.15) and (3.19).

If $F \in \mathcal{L} \cap \boldsymbol{\Phi}$, (3.9) follows by using the properties of the class $\mathcal{L}$ to establish analogies of relations (3.12) and (3.17). This ends the proof of the lemma.

Proof of Theorem 2.2. We use the idea in the proof of Theorem 2.2 of Wang [1] (e.g., Theorem 2.1 of this paper) to prove this result. Clearly, $F \in \Phi$ and $\mu<\infty$ imply $J_{F}^{+} \geq 1$. By (2.8), we have for any $\epsilon>0$, there exists some positive integer $n_{1}=n_{1}(T, \epsilon)$ such that

$$
\mathrm{E}(N(T))^{p} \mathbf{1}_{\left\{N(T)>n_{1}\right\}} \leq \epsilon
$$

To estimate the upper bound of $\Psi(x, T)$, we split it into two parts as

$$
\begin{aligned}
\Psi(x, T) & \leq \mathrm{P}\left(\sum_{j=1}^{N(T)} X_{j} e^{-\delta \tau_{j}}>x\right) \\
& =\left(\sum_{k=1}^{n_{1}}+\sum_{k=n_{1}+1}^{\infty}\right) \mathrm{P}\left(\sum_{j=1}^{k} X_{j} e^{-\delta \tau_{j}}>x, N(T)=k\right):=I_{3}+I_{4} .
\end{aligned}
$$

According to Lemma 3.4 of this paper and Lemma 3.5 of Wang [1], we have for sufficiently large $x$,

$$
\begin{aligned}
I_{3} & \leq(1+\epsilon) L_{F}^{-1} \sum_{k=1}^{n_{1}} \sum_{j=1}^{k} \mathrm{P}\left(X_{j} e^{-\delta \tau_{j}}>x, N(T)=k\right) \\
& \leq(1+\epsilon) L_{F}^{-1} \sum_{j=1}^{\infty} \mathrm{P}\left(X_{j} e^{-\delta \tau_{j}}>x, N(T) \geq j\right) \\
& =(1+\epsilon) L_{F}^{-1} \sum_{j=1}^{\infty} \mathrm{P}\left(X_{j} e^{-\delta \tau_{j}}>x, \tau_{j} \leq T\right) \\
& =(1+\epsilon) L_{F}^{-1} \int_{0-}^{T} \bar{F}\left(x e^{\delta t}\right) \lambda(\mathrm{d} t) .
\end{aligned}
$$


By Lemma 3.3, $F \in \Phi$, Lemma 3.1(ii), (3.20), and $p>J_{F}^{+} \geq 1$, there exists some positive constant $M$ such that for sufficiently large $x$,

$$
\begin{aligned}
I_{4} & \leq \sum_{k=n_{1}+1}^{\infty} \mathrm{P}\left(\sum_{j=1}^{k} X_{j}>x\right) \mathrm{P}(N(T)=k) \\
& \leq \bar{F}\left(p^{-1} x\right) \sum_{k=n_{1}+1}^{\infty} k \mathrm{P}(N(T)=k)+M(e \mu)^{p} x^{-p} \sum_{k=n_{1}+1}^{\infty} k^{p} \mathrm{P}(N(T)=k) \\
& \leq M \bar{F}(x)\left(\mathrm{EN}(T) \mathbf{1}_{\left\{N(T)>n_{1}\right\}}+\mathrm{E}(N(t))^{p} \mathbf{1}_{\left\{N(T)>n_{1}\right\}}\right) \\
& =M \epsilon \bar{F}(x) .
\end{aligned}
$$

By Lemma 3.1(i), for any $\gamma>J_{F}^{+}$, there exists some positive constant $a$ such that for sufficiently large $x$,

$$
\begin{aligned}
\int_{0-}^{T} \bar{F}\left(x e^{\delta t}\right) \lambda(\mathrm{d} t) & \geq a^{-1} \bar{F}(x) \int_{0-}^{T} e^{-\gamma \delta t} \lambda(\mathrm{d} t) \\
& \geq a^{-1} e^{-\gamma \delta T}(\lambda(T)-\lambda(0)) \bar{F}(x),
\end{aligned}
$$

which, combining (3.23) and $\lambda(T)-\lambda(0)>0$, implies

$$
I_{4} \leq M \epsilon \int_{0-}^{T} \bar{F}\left(x e^{\delta t}\right) \lambda(\mathrm{d} t)
$$

From (3.21), (3.22), and (3.25), we derive the right-hand side of (2.9).

As for the lower bound of $\Psi(x, T)$, by Lemma 3.4, we have for the above given $\epsilon>0$ and sufficiently large $x$,

$$
\begin{aligned}
\Psi(x, T) & \geq \mathrm{P}\left(\sum_{j=1}^{N(T)} X_{j} e^{-\delta \tau_{j}}>x+\int_{0}^{T} e^{-\delta s} C(\mathrm{~d} s)\right) \\
& \geq \sum_{k=1}^{n_{1}} \mathrm{P}\left(\sum_{j=1}^{k} X_{j} e^{-\delta \tau_{j}}>x+\int_{0}^{T} e^{-\delta s} C(\mathrm{~d} s), N(T)=k\right)
\end{aligned}
$$




$$
\begin{aligned}
& \geq(1-\epsilon) L_{F} \sum_{k=1}^{n_{1}} \sum_{j=1}^{k} \mathrm{P}\left(X_{j} e^{-\delta \tau_{j}}>x, N(T)=k\right) \\
& =(1-\epsilon) L_{F}\left(\sum_{j=1}^{\infty} \mathrm{P}\left(X_{j} e^{-\delta \tau_{j}}>x, \tau_{j} \leq T\right)-\sum_{k=n_{1}+1}^{\infty} \sum_{j=1}^{k} \mathrm{P}\left(X_{j} e^{-\delta \tau_{j}}>x, N(T)=k\right)\right) \\
& :=(1-\epsilon) L_{F}\left(\int_{0-}^{T} \bar{F}\left(x e^{\delta t}\right) \lambda(\mathrm{d} t)-I_{5}\right) .
\end{aligned}
$$

Analogously to the estimate for $I_{4}$, we have for sufficiently large $x$,

$$
\begin{aligned}
I_{5} & \leq \bar{F}(x) \operatorname{EN}(T) \mathbf{1}_{\left\{N(T)>n_{1}\right\}} \\
& \leq M \epsilon \int_{0-}^{T} \bar{F}\left(x e^{\delta t}\right) \lambda(\mathrm{d} t) .
\end{aligned}
$$

From (3.26) and (3.27), we obtain the left-hand side of (2.9).

If $F \in \mathcal{L} \cap \Phi$, then (2.4) follows by using (3.9) in the proof of (3.22) and (3.26).

\section{Numerical Calculations}

In this section, we perform some numerical calculations to check the accuracy of the asymptotic relations obtained in Theorem 2.2. The main work is to estimate the finite-time ruin probability defined in (1.2).

We assume that the claim sizes $\left\{X_{n}, n \geq 1\right\}$ come from the common Pareto distribution with parameter $\kappa=1, \beta=2$,

$$
F(x ; \kappa, \beta)=1-\left(\frac{\kappa}{\kappa+x}\right)^{\beta}, \quad x \geq 0,
$$

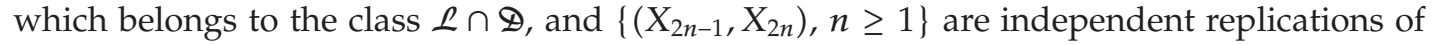
$\left(X_{1}, X_{2}\right)$ with the joint distribution

$$
F_{X_{1}, X_{2}}(x, y)=-\frac{1}{\alpha} \ln \left(1+\frac{\left(e^{-\alpha F(x)}-1\right)\left(e^{-\alpha F(y)}-1\right)}{e^{-\alpha}-1}\right)
$$

with parameter $\alpha=1$, where the joint distribution $F_{X_{1}, X_{2}}(x, y)$ is constructed according to the Frank Copula. It has been proved in Example 4.2 of Liu [12] that $X_{1}$ and $X_{2}$ are END r.v.s. Since $\left\{\left(X_{2 n-1}, X_{2 n}\right), n \geq 1\right\}$ are independent copies of $\left(X_{1}, X_{2}\right)$, the r.v.s $\left\{X_{n}, n \geq 1\right\}$ are END as well.

Assume that the claim arrival process $N(t)$ is the homogeneous Poisson process with intensity parameter $\lambda$. Clearly, such an integer-valued process $N(t)$ satisfies the condition (2.8). Choose $\lambda=0.1$. The total amount of premiums is simplified as $C(t)=c t$ with 
Table 1: Comparison between the analog value and the theoretical result in Theorem 2.2.

\begin{tabular}{lcc}
\hline$x\left(\times 10^{3}\right)$ & Theoretical result & Analog value \\
\hline 0.5 & $3.2846 e-6$ & $3.8120 e-6(16.1 \%)$ \\
1 & $8.2270 e-7$ & $9.1100 e-7(10.7 \%)$ \\
2 & $2.0586 e-7$ & $2.2300 e-7(8.3 \%)$ \\
5 & $3.2956 e-8$ & $3.5000 e-8(6.2 \%)$ \\
\hline
\end{tabular}

the premium rate $c=500$, and the constant interest rate $\delta=0.02$. Here, we set the time $T$ as $T=10$ and the initial capital reserve $x=500,10^{3}, 2 \times 10^{3}, 5 \times 10^{3}$, respectively. We aim to verify the accuracy of relation (2.4). The procedure of the computation of the finite-time ruin probability $\Psi(x, T)$ in Theorem 2.2 is listed here.

Step 1. Assign a value for the variable $x$ and set $l=0$.

Step 2. Divide the close interval $[0, T]$ into $m=1000$ pieces, and denote each time point as $t_{i}$, $i=1, \ldots, m$.

Step 3. For each $t_{i}$, generate a random number $n_{i}$ from the Poisson distribution $P\left(\lambda t_{i}\right)$, and set $n_{i}$ as the sample size of the claims.

Step 4 . Generate the accident arrival time $\left\{\tau_{k}^{i}, k=1, \ldots, n_{i}\right\}$ from the uniform distribution $U\left(0, t_{i}\right)$ and the claim sizes $\left\{X_{k^{\prime}}^{i} k=1, \ldots, n_{i}\right\}$ from (4.1) and (4.2).

Step 5. Calculate the expression $D$ below for each $t_{i}$ and denote them as $\left\{D_{i}, i=1, \ldots, m\right\}$ :

$$
D_{i}=\sum_{k=1}^{n_{i}} X_{k}^{i} e^{-r \tau_{k}^{i}}-\int_{0}^{t_{i}} e^{-r s} C(\mathrm{~d} s), \quad i=1, \ldots, m
$$

where $r$ and $C(t)$ have been defined and their values have also been assigned.

Step 6. Select the maximum value from $\left\{D_{i}, i=1, \ldots, m\right\}$, and denote it as $D^{*}$, compare $D^{*}$ with $x$; if $D^{*}>x$, then the value of $l$ increases 1 .

Step 7. Repeat Step 2 through Step 6, $N=10^{9}$ times.

Step 8. Calculate the moment estimate of the finite-time ruin probability, $l / N$.

Step 9. Repeat Step 1 through Step 8 ten times and get ten estimates. Then, choose the median of the ten estimates as the analog value of the finite-time ruin probability.

For different value of $x$, the analog value and the theoretical result of the finite-time ruin probability are presented in Table 1 , and the percentage of the error relative to the theoretical result is also presented in the bracket behind the analog value. It can be found that from Table 1, the larger $x$ becomes, the smaller the difference between the analog value and the theoretical result is. Therefore, the approximate relationship in Theorem 2.2 is reasonable. 


\section{Acknowledgments}

The authors would like to thank the two referees for their useful comments on an earlier version of this paper. The revision of this work was finished during a research visit of the first author to Vilnius University. He would like to thank the Faculty of Mathematics and Informatics for its excellent hospitality. Research supported by National Natural Science Foundation of China (no. 11001052), China Postdoctoral Science Foundation (20100471365), National Science Foundation of Jiangsu Province of China (no. BK2010480), Natural Science Foundation of the Jiangsu Higher Education Institutions of China (no. 09KJD110003), Postdoctoral Research Program of Jiangsu Province of China (no. 0901029C), and Jiangsu Government Scholarship for Overseas Studies, Qing Lan Project.

\section{References}

[1] D. Wang, "Finite-time ruin probability with heavy-tailed claims and constant interest rate," Stochastic Models, vol. 24, no. 1, pp. 41-57, 2008.

[2] D. B. H. Cline, "Intermediate regular and $\Pi$ variation," Proceedings of the London Mathematical Society, vol. 68, no. 3, pp. 594-616, 1994.

[3] P. Embrechts and E. Omey, "A property of longtailed distributions," Journal of Applied Probability, vol. 21, no. 1, pp. 80-87, 1984 .

[4] N. H. Bingham, C. M. Goldie, and J. L. Teugels, Regular Variation, Cambridge University Press, Cambridge, UK, 1987.

[5] Q. Tang and G. Tsitsiashvili, "Precise estimates for the ruin probability in finite horizon in a discretetime model with heavy-tailed insurance and financial risks," Stochastic Processes and their Applications, vol. 108, no. 2, pp. 299-325, 2003.

[6] C. Klüppelberg and U. Stadtmüller, "Ruin probabilities in the presence of heavy-tails and interest rates," Scandinavian Actuarial Journal, no. 1, pp. 49-58, 1998.

[7] S. Asmussen, "Subexponential asymptotics for stochastic processes: extremal behavior, stationary distributions and first passage probabilities," The Annals of Applied Probability, vol. 8, no. 2, pp. 354374, 1998.

[8] V. Kalashnikov and D. Konstantinides, "Ruin under interest force and subexponential claims: a simple treatment," Insurance: Mathematics and Economics, vol. 27, no. 1, pp. 145-149, 2000.

[9] D. Konstantinides, Q. Tang, and G. Tsitsiashvili, "Estimates for the ruin probability in the classical risk model with constant interest force in the presence of heavy tails," Insurance: Mathematics and Economics, vol. 31, no. 3, pp. 447-460, 2002.

[10] Q. Tang, "Asymptotic ruin probabilities of the renewal model with constant interest force and regular variation," Scandinavian Actuarial Journal, no. 1, pp. 1-5, 2005.

[11] Q. Tang, "The finite-time ruin probability of the compound Poisson model with constant interest force," Journal of Applied Probability, vol. 42, no. 3, pp. 608-619, 2005.

[12] L. Liu, "Precise large deviations for dependent random variables with heavy tails," Statistics $\mathcal{E}$ Probability Letters, vol. 79, no. 9, pp. 1290-1298, 2009.

[13] N. Ebrahimi and M. Ghosh, "Multivariate negative dependence," Communications in Statistics, vol. 10, no. 4, pp. 307-337, 1981.

[14] H. W. Block, T. H. Savits, and M. Shaked, "Some concepts of negative dependence," The Annals of Probability, vol. 10, no. 3, pp. 765-772, 1982.

[15] Q. Tang and G. Tsitsiashvili, "Randomly weighted sums of subexponential random variables with application to ruin theory," Extremes, vol. 6, no. 3, pp. 171-188, 2003.

[16] Y. Chen, A. Chen, and K. W. Ng, "The strong law of large numbers for extended negatively dependent random variables," Journal of Applied Probability, vol. 47, no. 4, pp. 908-922, 2010.

[17] Q. Tang, "Insensitivity to negative dependence of the asymptotic behavior of precise large deviations," Electronic Journal of Probability, vol. 11, pp. 107-120, 2006.

[18] Y. Chen and K. C. Yuen, "Sums of pairwise quasi-asymptotically independent random variables with consistent variation," Stochastic Models, vol. 25, no. 1, pp. 76-89, 2009. 


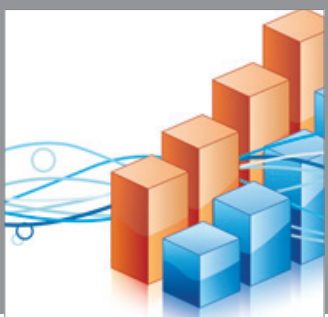

Advances in

Operations Research

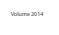

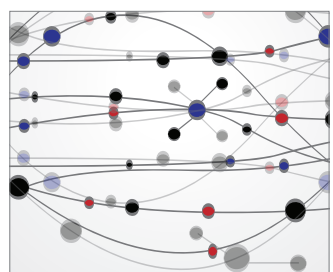

\section{The Scientific} World Journal
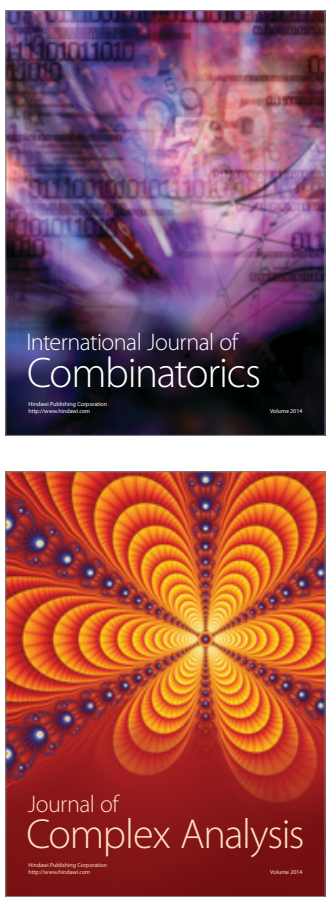

International Journal of

Mathematics and

Mathematical

Sciences
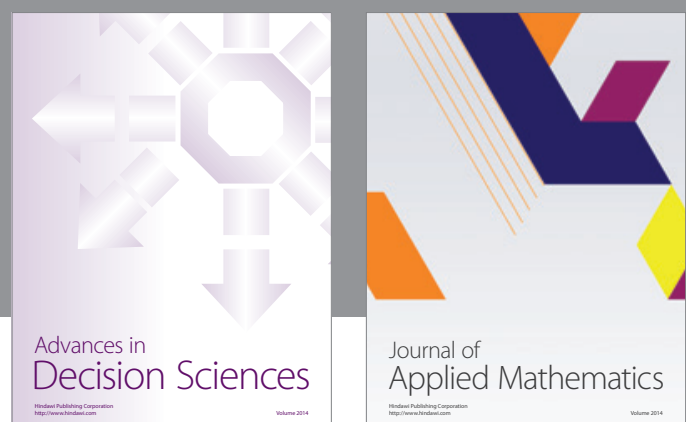

Journal of

Applied Mathematics
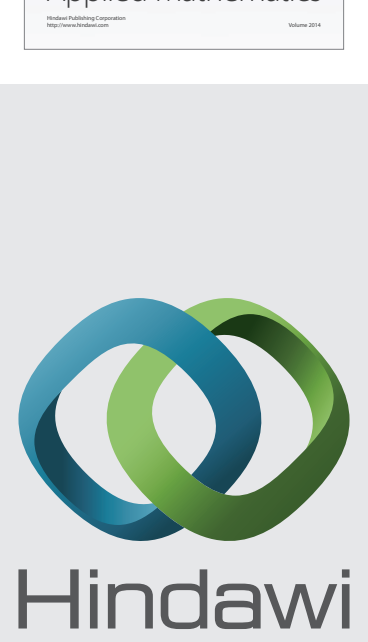

Submit your manuscripts at http://www.hindawi.com
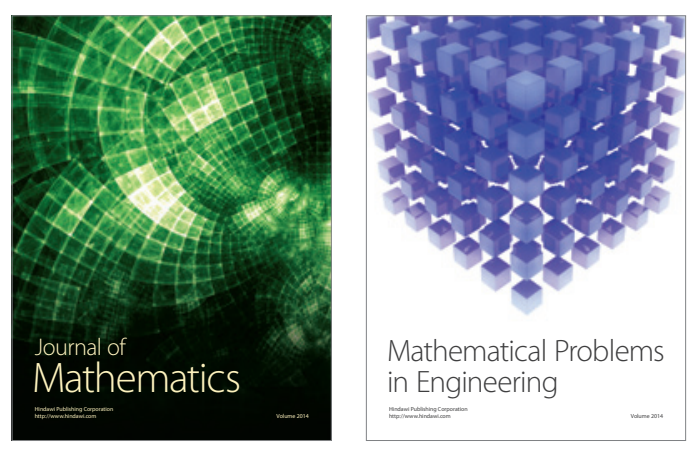

Mathematical Problems in Engineering
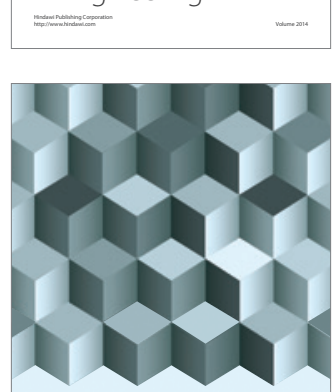

Journal of

Function Spaces
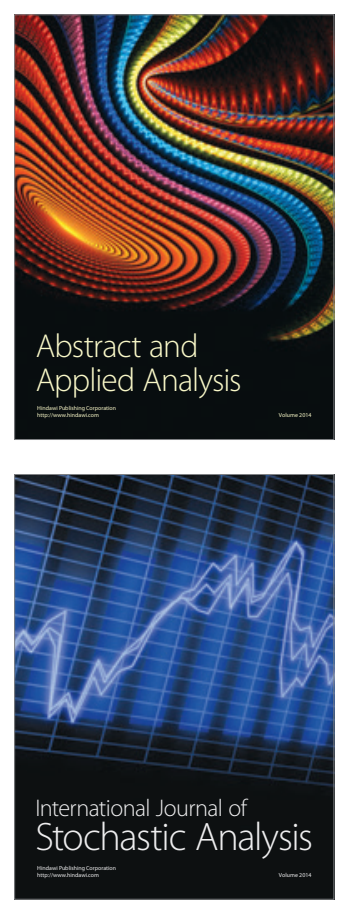

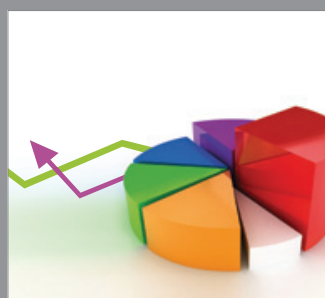

ournal of

Probability and Statistics

Promensencen
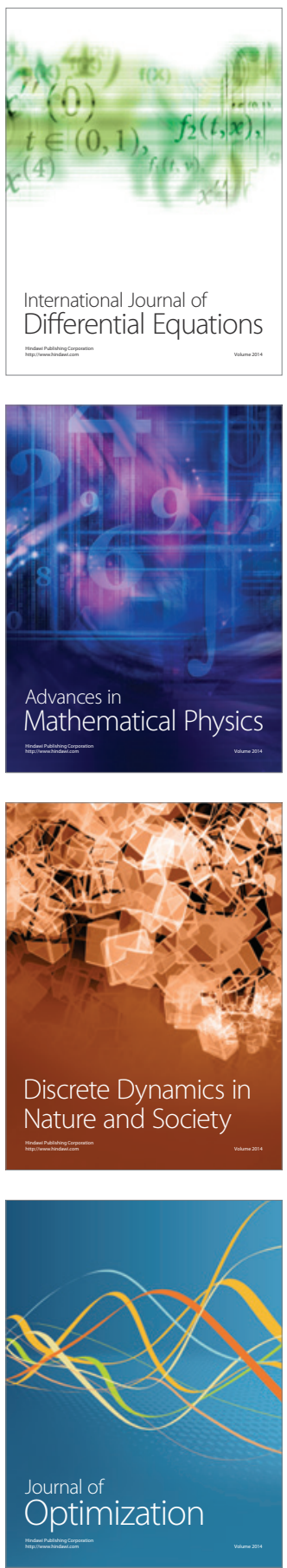